\title{
Phenomena in rank-one $\mathbb{Z}^{2}$-actions
}

\author{
by \\ Tomasz Downarowicz and Jacek Serafin (Wrocław)
}

\begin{abstract}
We present an example of a rank-one partially mixing $\mathbb{Z}^{2}$-action which possesses a non-rigid factor and for which the Weak Closure Theorem fails. This is in sharp contrast to one-dimensional actions, which cannot display this type of behavior.
\end{abstract}

1. Introduction. The centralizer of an abelian group of measure-preserving transformations (measure-preserving action, for short) has long been an object of extensive study in ergodic theory. It is defined as the family of all automorphisms of the underlying space, commuting with all elements of the action.

Rank-one systems constitute an important class of measure-preserving transformations; their behavior can be nicely expressed in terms of cutting and stacking of one tower. A deep and fundamental result of King ([2]), which is known as the Weak Closure Theorem, brings the above notions together and refers to $\mathbb{Z}$-actions (i.e. one-dimensional actions induced by powers of a single automorphism): if a $\mathbb{Z}$-action is rank-one, then its centralizer is the weak closure of the powers of the transformation.

We will say that the action satisfies the Weak Closure Theorem (WCT for short) if its centralizer equals the weak closure of the elements of the action.

A natural question arises whether WCT holds for more general actions than $\mathbb{Z}$-actions. Downarowicz and Kwiatkowski ([1]) answered this question negatively, providing an example of a rank-one $\mathbb{Z}^{2}$-action for which WCT fails. Their example was in the form of a Morse flow, so it was constructed in the absence of mixing. Subsequently, Jean-Paul Thouvenot suggested that perhaps rank-one $\mathbb{Z}^{2}$-actions satisfying an additional condition of mild mixing should obey WCT. In this note we prove that this is not the case, even if one imposes a stronger mixing condition, called partial mixing.

2000 Mathematics Subject Classification: 37A05, 37A15, 37A35.

Key words and phrases: rank-1, partial mixing, centralizer. 
The existence or nonexistence of factors with certain properties is another issue where it turns out that the dimension of the acting group plays a crucial role. It can be deduced from King's theorem that if a rank-one $\mathbb{Z}$-action has a nontrivial factor, then this factor has to be rigid, and consequently the action cannot be mildly mixing. We show that a $\mathbb{Z}^{2}$-action can be rank-one, mildly mixing and can possess a nonrigid factor.

2. Preliminaries and notation. Let $\mathbb{Z}^{2}$ denote the set of 2-dimensional integers $\bar{n}=\left(n_{h}, n_{v}\right)$; the first (horizontal) coordinate is the column number increasing to the right, the second (vertical) is the row number, increasing upward (unlike the usual notation of matrices, but as in the Cartesian coordinate system); $\overline{0}=(0,0), \overline{1}=(1,1)$. We write $\bar{m} \leq \bar{n}$ whenever $m_{h} \leq n_{h}$ and $m_{v} \leq n_{v}$. For $\bar{m} \leq \bar{n}$ the rectangle $\{\bar{i}: \bar{m} \leq \bar{i} \leq \bar{n}\}$ is denoted by $[\bar{m}, \bar{n}]$.

Let $A$ be a finite set. A finite $A$-valued matrix $B=(B(\bar{i}))_{\bar{i} \in[\overline{1}, \bar{n}]}$ is called a block of size $\bar{n}$. For two blocks $B$ and $C$ of the same size $\bar{n}$ the Hamming distance is defined as

$$
\bar{d}(B, C)=\frac{\#\{\bar{i} \in[\overline{1}, \bar{n}]: B(\bar{i}) \neq C(\bar{i})\}}{\#[\overline{1}, \bar{n}]} .
$$

The elements of $A^{\mathbb{Z}^{2}}$ are called arrays. For an array $x=(x(\bar{n}))$, the block $B(\bar{i})=x(\bar{m}+\bar{i}-\overline{1})(\bar{i} \in[\overline{1}, \bar{n}])$ will be denoted by $x[\bar{m}, \bar{m}+\bar{n}-\overline{1}]$ and we will say that $B$ occurs in $x$ at position $\bar{m}$. Similarly, a block $B$ may occur in another block $C$ (larger in size). The Hamming distance between arrays $x$ and $y$ is defined as the upper limit of the distances of the blocks $x[-\bar{n}, \bar{n}]$ and $y[-\bar{n}, \bar{n}]$ when both coordinates of $\bar{n}$ tend to infinity.

We will consider the $\mathbb{Z}^{2}$-action of the group $\mathbf{T}=\left\{T^{\bar{n}}: \bar{n} \in \mathbb{Z}^{2}\right\}$ of shifts on $A^{\mathbb{Z}^{2}}$ defined by

$$
T^{\bar{n}} x(\bar{m})=x(\bar{m}+\bar{n}) .
$$

Let $(X, \mu, \mathbf{T})$ be a measure preserving $\mathbb{Z}^{2}$-action of the shifts on arrays over a finite alphabet $A$. We now recall what it means that $(X, \mu, \mathbf{T})$ is of rank one.

If $x$ is an array, then we say that a block $B$ of size $\bar{n} \varepsilon$-occurs in $x$ at position $\bar{m}$ if

$$
\bar{d}(B, x[\bar{m}, \bar{m}+\bar{n}-\overline{1}]) \leq \varepsilon .
$$

Suppose that $B$ is a block of size $\bar{n}$. We say that $B \varepsilon$-covers $x$ if there exists a subset $\mathbf{P} \subset \mathbb{Z}^{2}$ with the following properties:

(1) $\#\left(\left[\bar{m}_{1}, \bar{m}_{1}+\bar{n}-\overline{1}\right] \cap\left[\bar{m}_{2}, \bar{m}_{2}+\bar{n}-\overline{1}\right]\right) \leq \varepsilon n_{h} n_{v}$ for all $\bar{m}_{1}, \bar{m}_{2} \in \mathbf{P}$,

(2) the density of $\mathbf{P}$ in $\mathbb{Z}^{2}$ is at least $(1-\varepsilon) / n_{h} n_{v}$,

(3) $B \varepsilon$-occurs in $x$ at every position $\bar{m} \in \mathbf{P}$.

Informally, some almost disjoint $\varepsilon$-occurrences of $B$ in $x$ cover nearly all of $\mathbb{Z}^{2}$. 
REMARK 1. Classically, one requires in (1) that the rectangular regions are disjoint. The version we propose is equivalent; the regions become disjoint after slightly trimming the margins of the covering block.

The system $(X, \mu, \mathbf{T})$ is rank-one if for every $\varepsilon>0$ there exists a block $B_{\varepsilon}$ such that $\mu$-almost every $x \in X$ can be $\varepsilon$-covered by $B_{\varepsilon}$, and both dimensions of the covering blocks tend to infinity as $\varepsilon \rightarrow 0$.

The centralizer $\mathcal{C}(X, \mu, \mathbf{T})$ of $(X, \mu, \mathbf{T})$ is the set of all automorphisms of the Borel measure space $(X, \mu)$ which commute with every element of the acting group $\mathbf{T}$. Obviously, $\mathbf{T} \subset \mathcal{C}(X, \mu, \mathbf{T})$. If $\mathcal{C}(X, \mu, \mathbf{T})$ is equipped with the weak topology defined as follows:

$$
\begin{aligned}
S_{n} \rightarrow S \Leftrightarrow & \text { for every Borel set } F \subset X, \\
& \mu\left(S_{n}(F) \triangle S(F)\right) \rightarrow 0 \text { and } \mu\left(S_{n}^{-1}(F) \triangle S^{-1}(F)\right) \rightarrow 0,
\end{aligned}
$$

then it becomes a complete metric topological group. The closure of $\mathbf{T}$ in $\mathcal{C}(X, \mu, \mathbf{T})$ in the weak topology is denoted by $W c l(\mathbf{T})$. Clearly, if the centralizer of $\mathbf{T}$ equals $\operatorname{Wcl}(\mathbf{T})$, then all the elements of $\mathcal{C}(X, \mu, \mathbf{T})$ can be weakly approximated by elements of the group acting on $X$.

We recall that a transformation $T$ is mildly mixing if there are no nontrivial rigid functions, i.e. if $f \in L^{2}(X)$ and there exists a sequence $\bar{n}_{k} \rightarrow \infty$ such that $T^{\bar{n}_{k}} f \rightarrow f$ in $L^{2}(X)$ then $f$ is a constant function.

Another useful concept is partial mixing. A transformation $T$ is called partially mixing if there exists a positive constant $\alpha$ such that for every pair of measurable sets $A, B$ of positive measure,

$$
\lim \inf \mu\left(A \cap T^{\bar{n}} B\right) \geq \alpha \mu(A) \mu(B) .
$$

We close this section by mentioning that partial mixing implies mild mixing, and mild mixing implies weak mixing.

3. Main result. Our goal is to prove the following theorem:

THEOREM 1. There exists an ergodic, rank-one, measure-preserving $\mathbb{Z}^{2}$ action $(Y, \nu, \mathbf{T})$ with the following properties: it is partially mixing, has a nonrigid factor, and does not obey WCT.

REMARK 2.

1. Mildly mixing, rank-one $\mathbb{Z}$-actions have no nontrivial factors ([2]) (and obey WCT).

2. Our construction is a modification of that in [1]. The $\mathbb{Z}^{2}$-action is a finite group extension of a rank-one base system. The structure of the base system is very similar to that in [1], but by introducing a random fluctuation of positioning of some blocks we ensure that the system is partially mixing. 
3. It is worth noticing that Ryzhikov [3] proved that our example is optimal in the following sense: if a rank-one $\mathbb{Z}^{d}$-action has a nonrigid factor then it is a finite extension of this factor (a finite extension is always a group extension).

In the course of the proof we shall need a general statement concerning the existence of long one-dimensional blocks with good randomness properties, the entries of the block taking values in a finite group.

LEMma 1 (see [1] for a similar argument). Fix a finite group $G$ and let $m$ denote its order. For every $\varepsilon>0$ there exists $q \in \mathbb{N}$ such that if $n \geq q$ then there exists a vector $V=\left[v_{1}, \ldots, v_{n}\right]$ of length $n$ over $G$ such that for every $0<k \leq n / 2$ and $g \in G$,

$$
\left|\frac{\#\left\{1 \leq i \leq n-k: v_{i}=g\right\}}{n-k}-\frac{1}{m}\right|<\varepsilon
$$

and

$$
\left|\frac{\#\left\{1 \leq i \leq n-k: v_{i+k} v_{i}^{-1}=g\right\}}{n-k}-\frac{1}{m}\right|<\varepsilon .
$$

Proof. Consider an i.i.d. sequence $X_{1}, X_{2}, \ldots$ of random variables taking values in $G$ with the uniform distribution $(1 / m, 1 / m, \ldots, 1 / m)$. Note that for any positive integer $k$ the sequence $X_{i+k} X_{i}^{-1}(i=1,2, \ldots)$ is also i.i.d. Fix $g \in G$ and let $Y_{i}=1$ whenever $X_{i}=g$, and $Y_{i}=0$ otherwise. Similarly, let $Y_{i}^{\prime}=1$ whenever $X_{i+k} X_{i}^{-1}=g$, and zero otherwise. Now, $\left(Y_{i}\right)$ and $\left(Y_{i}^{\prime}\right)$ are both i.i.d. sequences of Bernoulli trials with probability of success $1 / \mathrm{m}$. Applying the central limit theorem we easily estimate that

$$
\mathbb{P}\left(\left|\frac{1}{n-k} \sum_{i=1}^{n-k} Y_{i}-\frac{1}{m}\right|>\varepsilon\right) \leq 2 e^{-C_{\varepsilon} \sqrt{n-k}}
$$

(and the same for $Y_{i}^{\prime}$ ), where $C_{\varepsilon}$ is a positive constant (depending only on $\varepsilon$ ). Thus, summing over $k=0,1, \ldots, n / 2$, we see that the probability that for at least one such $k$ the above absolute value involving $Y_{i}$ or the one involving $Y_{i}^{\prime}$ exceeds $\varepsilon$, is estimated by $4(n+2) e^{-C_{\varepsilon} \sqrt{n / 2}}$, which, for $n$ sufficiently large (larger than some $q$ ), is smaller than 1 . Thus the set of $G$-valued vectors $V$ of length $n$ satisfying the required conditions is nonempty.

4. The base process. The base of the skew product will act on arrays over the alphabet $A=\{0,1\}$. We construct inductively an ascending sequence $\left\{B_{t}\right\}_{t \geq 0}$ of square blocks, consisting of 0 's and 1's; those blocks 
define the base of our skew product transformation. We also introduce an auxiliary sequence of blocks $\left\{C_{t}\right\}$.

For a block $B$, let $B^{k \times l}$ denote the concatenation of $k \cdot l$ copies of $B$, arranged in a block matrix with $k$ columns and $l$ rows of copies of $B$. Fix also a summable sequence of positive numbers $\varepsilon_{t}$. Finally, let $r \geq 2$ be a fixed positive integer.

As the inductive procedure refers to two preceding steps, steps 0 and 1 will be the initial steps: put $n_{-1}=0, n_{0}=1$ and let $B_{0}$ be the $1 \times 1$ matrix with entry 1 . Let $C_{0}=B_{0}^{r \times r}$, i.e., the square block of size $r n_{0} \times r n_{0}$ filled entirely with the symbols 1 . Pick some $q_{1}>1$ and let $B_{1}=C_{0}^{6 q_{1} \times 6 q_{1}}$. Note that the size of $B_{1}$ is $n_{1} \times n_{1}$, where $n_{1}=6 q_{1} r n_{0}$.

Suppose that $B_{t}$ is already defined and has size $n_{t} \times n_{t}$ and that $n_{t-1} \geq 1$ is known and much smaller than $n_{t}$. We set $C_{t}=B_{t}^{r \times r}$. Now we apply Lemma 1 with $G=\mathbb{Z}_{r n_{t-1}} \times \mathbb{Z}_{r n_{t-1}}$ and $\varepsilon=\varepsilon_{t}$ to obtain a number $q$ and two "random" vectors, denoted by $H=\left\{\bar{h}_{i}\right\}$ and $V=\left\{\bar{v}_{i}\right\}$, of lengths $2 q$ and $q$, respectively. We set $q_{t+1}=q$ and

$$
n_{t+1}=6 q_{t+1} r n_{t} .
$$

We need to define $B_{t+1}$ of size $n_{t+1} \times n_{t+1}$. We begin by dividing the (temporarily empty) $n_{t+1} \times n_{t+1}$ square region in $\mathbb{Z}^{2}$ into some "windows", as follows: the left half of this square is divided into $2 q_{t+1}$ horizontal windows (denoted $\mathcal{H}_{i}, i=1, \ldots, 2 q_{t+1}$ ) of size $3 q_{t+1} r n_{t} \times 3 r n_{t}$. The right half is divided into $q_{t+1}$ vertical windows (denoted $\mathcal{V}_{i}, i=1, \ldots, q_{t+1}$ ) of size $3 r n_{t} \times 6 q_{t+1} r n_{t}$ (see Figure 1).

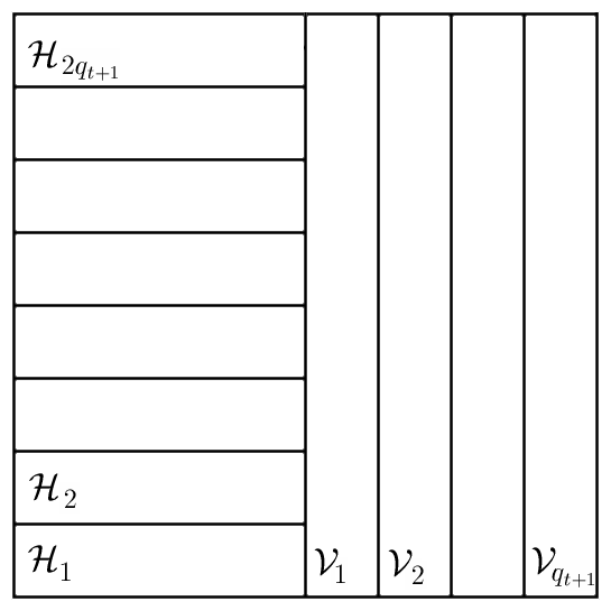

Fig. 1. Windows in the square region 
Recall now that $C_{t}$ is a square of size $r n_{t}$, so a concatenation $C_{t}^{3 q_{t+1} \times 3}$ is a block, which we call a horizontal bar, of size $3 q_{t+1} r n_{t} \times 3 r n_{t}$, which is the exact size of the horizontal window. We put this horizontal bar inside every horizontal window $\mathcal{H}_{i}$, for $i=1, \ldots, 2 q_{t+1}$. Next we shove the bar placed inside the window $\mathcal{H}_{i}$ by the "random" vector $\bar{h}_{i}$ (this is the $i$ th entry of $H$ and it is an element of $\mathbb{Z}_{r n_{t-1}} \times \mathbb{Z}_{r n_{t-1}}$ ) regarded as a nonnegative element of $\mathbb{Z}^{2}$. This operation brings a small (relative to the size of the bar) portion of the horizontal bars (at most $r n_{t-1}$ of the top rows and $r n_{t-1}$ of the rightmost columns of the bar) outside the windows $\mathcal{H}_{i}$. We trim this part of the bar. At the bottom and in the left side of the window, empty spaces will appear, which we fill with zeros.

Similarly, we define a vertical bar as a concatenation $C_{t}^{3 \times 6 q_{t+1}}$, and we place this bar inside every vertical window of the right half of the square region. Then, for each $i=1, \ldots, q_{t+1}$, we shove by $\bar{v}_{i}$ the bar inside the window $\mathcal{V}_{i}$ (and trim the result). As before, we fill the empty spaces with zeros.

This completes the definition of our block $B_{t+1}$ (see Figure 2 ; the empty spaces filled with zeros are marked black).

The base system is now defined as a measure-preserving $\mathbb{Z}^{2}$-action $(X, \mu, \mathbf{T})$; if $B$ is a block, then $\mu(B)$ is the limiting frequency of occurrences of $B$ in the blocks $B_{t}$; this limit exists as a simple consequence of the covering property of the blocks $B_{t}$.

5. The finite extension. Let $\mathbb{Z}_{r}$ denote the cyclic group of order $r$. We now define a new system $(Y, \nu, \mathbf{T})$, which is an ergodic $\mathbb{Z}_{r}$-extension of the base process $(X, \mu, \mathbf{T})$, described in the preceding section. We need to define a cocycle $\Phi: \mathbb{Z}^{2} \times X \rightarrow \mathbb{Z}_{r}$ so that

$$
\Phi(\bar{n}+\bar{m}, x)=\Phi(\bar{n}, x)+\Phi\left(\bar{m}, T^{\bar{n}} x\right) .
$$

Then the skew product is defined classically by

$$
T^{\bar{n}}(x, g)=\left(T^{\bar{n}}(x), g+\Phi(\bar{n}, x)\right) .
$$

In order to define the cocycle, with each block $B_{t}$ we associate some $\mathbb{Z}_{r}$-valued array $G_{t}$ of the same size as $B_{t}$ in such a way that whenever $B_{t}$ (perhaps slightly trimmed) occurs somewhere in $B_{t+1}$ then $G_{t+1}$, restricted to the corresponding region, equals $G_{t}+z$ (trimmed appropriately) for some $z \in \mathbb{Z}_{r}$ ( $z$ is added to each entry of $G_{t}$ ). Once this is done, for $x \in X$ and $\bar{n} \in \mathbb{Z}^{2}$ we find $t$ large enough so that the positions $\overline{0}$ and $\bar{n}$ in $x$ are covered by the same copy of the block $B_{t}$ (such $t$ can be found except for points $x$ in a set of measure zero). Then the formula

$$
\Phi(\bar{n}, x)=G_{t}(\bar{m}+\bar{n})-G_{t}(\bar{m}),
$$




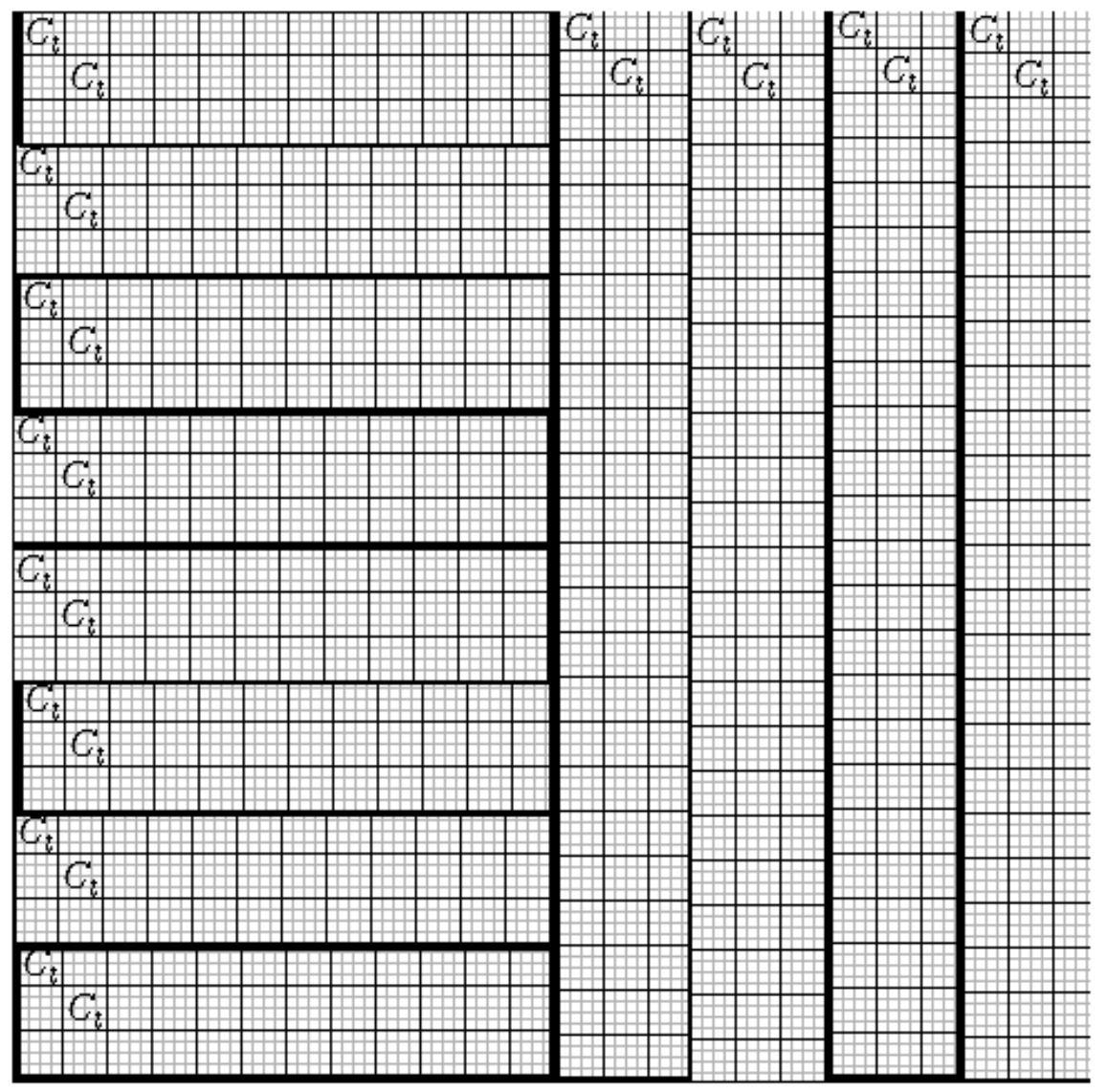

Fig. 2. The block $B_{t+1}$ for $r=4, q_{t+1}=4$. The smallest squares represent the blocks $B_{t}$. The larger squares are $C_{t}$. The "shoving vectors" should be much smaller than the size of $B_{t}$ (in the figure the shoving is oversized to make it visible).

where $\bar{m}, \bar{m}+\bar{n}$ are the relative coordinates in $B_{t}$ of $\overline{0}$ and $\bar{n}$ in $x$, respectively, is clearly a consistent definition of a measurable cocycle on $X$.

REMARK 3. It is well known that the finite extension can also be viewed as a shift system over the alphabet $\mathbb{Z}_{r}$. Almost every element of the system is a limit of the blocks $G_{t}+z$ expanding in all four directions around the origin as $t$ grows.

So, here is how we define $G_{t}$. First $G_{1}$ is the $1 \times 1$ matrix with entry $0 \in \mathbb{Z}_{r}$. Suppose that $G_{t}$ is already defined for some $t \in \mathbb{N}$. Recall that $B_{t}$ occurs in $B_{t+1}$ organized in blocks $C_{t}$. With $C_{t}$ we associate a matrix $D_{t}$ over the alphabet $\mathbb{Z}_{r}$ of the same size as $C_{t}$ : we concatenate the matrices $G_{t}+z$ starting with $z=0$ for the bottom-left copy and advancing $z$ by 1 each step 
to the right or upwards (now a "step" means a jump by $n_{t}$ coordinates). Note that in the bottom-right and top-left "corners" of $D_{t}$ (meaning a copy of $G_{t}+z$ occupying a corner), the parameter $z$ equals $r-1$, so if we concatenate another copy of $D_{t}$ above or on the right, the progression of the parameter $z$ will be maintained. Next we create horizontal and vertical bars as in the construction of $B_{t+1}$, but this time we concatenate copies of $D_{t}$ rather than $C_{t}$ (see Figure 3). Notice that for each $z \in \mathbb{Z}_{r}$, if we ignore a few columns on the left and on the right, the remaining part of the horizontal bar is a concatenation of copies of $D_{t}+z$ (an analogous statement holds for the vertical bar with a few rows ignored).

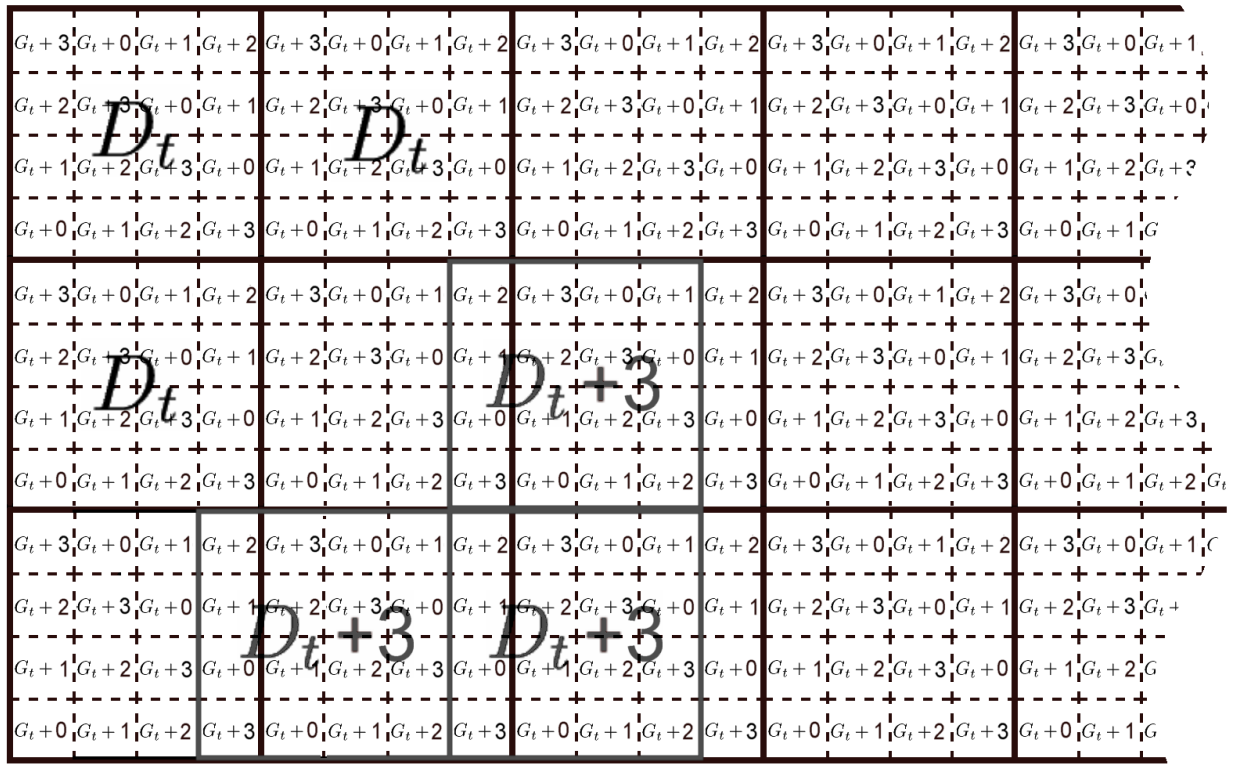

Fig. 3. The structure of the horizontal bar, component of $G_{t+1}$

With the $\mathbb{Z}_{r}$-valued bars ready, we can define $G_{t+1}$ by replacing in $B_{t+1}$ each horizontal and vertical bar by its $\mathbb{Z}_{r}$-valued counterpart, shoved and trimmed appropriately (just as we did in the process of defining $B_{t+1}$ ). At all remaining positions we put zeros. This concludes the definition of $G_{t+1}$.

One needs to realize that, in the symbolic representation, (almost) every element of the extension is covered, with high accuracy (for every $t$ ), by the blocks $G_{t+1}+z$ with $z$ ranging over $\mathbb{Z}_{r}$. Moreover, each $z$ occurs in this role with equal frequency. Thus the coordinatewise addition of a fixed element $z$ of $\mathbb{Z}_{r}$ is an automorphism of this system. 
It remains to prove that the extension is rank-one and partially mixing. Either property implies ergodicity of the extension, whereas the partial mixing property will prevent the aforementioned automorphism (addition of $z$ ) from being weakly approximated by the elements $T^{\bar{n}}$. This way we will have checked all the properties for which the system has been built.

We hence proceed to proving rank-one. As already noticed, almost each element is nearly covered by the blocks $G_{t+1}+z$, but it will be more convenient for us to consider $G_{t+1}-z$ instead. So, it suffices to show that every such block is almost covered by the copies of the same block $D_{t}$ already without adding $z$ to it. But this follows from the former observation that the horizontal and vertical bars (which cover $G_{t+1}$ with high accuracy), after appropriate truncation, become concatenations of copies of $D_{t}+z$. Clearly, the truncation influences the accuracy of the covering very little. Thus $G_{t+1}-z$ is well covered by copies $D_{t}$, as required, and the system is rank-one.

The remaining part is devoted to proving that $(Y, \nu, \mathbf{T})$ is partially mixing. Since for rank-one transformations the cylinders corresponding to the covering blocks (and their shifted images) generate, to prove partial mixing it suffices to show that for any $t \geq 1$, whenever $\bar{n}$ is sufficiently distant from $\overline{0}$ then

$$
\mu\left(\left[D_{t-1}\right] \mid T^{\bar{n}}\left[D_{t-1}\right]\right)>C \mu\left(\left[D_{t-1}\right]\right),
$$

where $C$ is a positive constant (we use the notation $[B]=\{x: x[\overline{1}, \bar{m}]=B\}$ for the cylinder corresponding to a block $B$ of size $\bar{m}$ ). We assume that both coordinates of $\bar{n}$ are nonnegative, the other three cases being symmetric. In order to prove that (2) holds for all large enough $\bar{n}$, we begin by showing that it is true for a large but fixed $t$ with the additional assumption that $\bar{n}=\left(n_{h}, n_{v}\right)$ satisfies the condition

$$
n_{t}-n_{t-1} \leq \max \left(n_{h}, n_{v}\right)<n_{t+1}-n_{t} .
$$

For a general sufficiently large $\bar{n}$ we then apply the following argument: Clearly, $\bar{n}$ satisfies, for some $s \geq t$,

$$
n_{s}-n_{s-1} \leq \max \left(n_{h}, n_{v}\right)<n_{s+1}-n_{s} .
$$

The block $D_{s-1}$ is (nearly) covered by copies of $D_{t-1}$, say $D_{t-1}$ occurs in relative coordinates in $D_{s-1}$ at positions $\bar{k}$, with both coordinates between 0 and $r n_{s-1}$. The corresponding cylinder $\left[D_{t-1}\right]$ is thus a disjoint union of the cylinders $\left[D_{s-1}\right]$ shifted by all the vectors $\bar{k}$. Thus the intersection $\left[D_{t-1}\right] \cap T^{\bar{n}}\left[D_{t-1}\right]$ can be written as the disjoint union of intersections of the form $T^{\bar{k}}\left[D_{s-1}\right] \cap T^{\bar{n}+\bar{k}^{\prime}}\left[D_{s-1}\right]$, which (when calculating the measure) can be replaced by $\left[D_{s-1}\right] \cap T^{\bar{n}+\bar{k}^{\prime}-\bar{k}}\left[D_{s-1}\right]$. It is now easy to see (by symmetry of the distribution of $\bar{k}^{\prime}-\bar{k}$ ) that at least a quarter of the exponents 
satisfy (4) (see Figure 4). It will follow that the inequality (2) holds for $D_{t-1}$ with a constant $C$ replaced by $C / 4$.

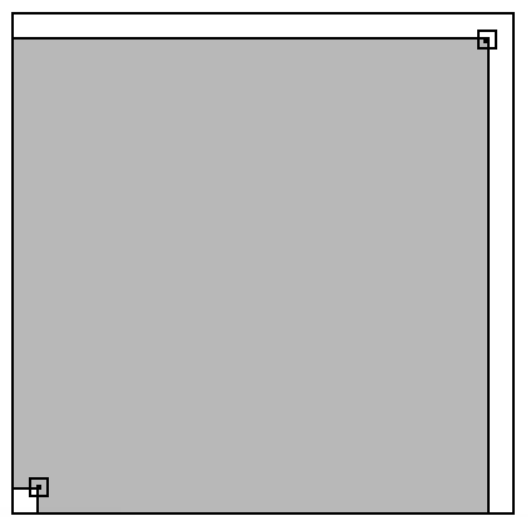

Fig. 4. The range of $\bar{n}$ satisfying (4) is marked gray. For every $\bar{n}$ in this range at least a quarter of the range of $\bar{n}+\bar{k}^{\prime}-\bar{k}$ (the small square) falls in the gray area. Two extreme cases are shown.

Let us now begin the proof of (2) (assuming (3)) by considering an $x \in Y$ and $y=T^{\bar{n}} x$. We need to estimate the density of the bottom-left corners of the copies of $D_{t-1}$ (we will call them SW-corners, for short) in $x$ matching $\mathrm{SW}$-corners in $y$. Because almost all of $x$ is covered by copies of the blocks $D_{t+1}$, we will restrict the count to one such copy. In the density count below we will ignore all small fractions resulting from the covering inaccuracies. These converge to zero as $t \rightarrow \infty$, and hence do not contribute to the rate of mixing.

Recall that $D_{t+1}$ in $x$ decomposes into jointly $r^{2}$ copies of $G_{t+1}+z$ (with varying $z$ ). Because both $n_{v}$ and $n_{h}$ are between zero and $n_{t+1}$ (the size of $\left.G_{t+1}\right)$, as many as $(r-1)^{2}$ of these copies fall completely inside the $D_{t+1}$ in $y$ (we will call them "good"). Every such good copy overlaps in $y$ with four or two (if $n_{h}=0$ or $n_{v}=0$ ) adjacent (with no spaces between them) copies of $G_{t+1}+z^{\prime}$, namely its bottom-left subrectangle overlaps with $G_{t+1}+z$, the bottom-right and top-left subrectangles overlap with $G_{t+1}+z+1$, and the top-right subrectangle overlaps with $G_{t+1}+z+2$ (see Figure 5).

We multiply the rate of partial mixing by a constant $(r-1)^{2} / r^{2}$ and we restrict our search to one "good" copy of $G_{t+1}+z$.

The positions of the four (or two) above mentioned copies of $G_{t+1}+z^{\prime}$ in $y$ differ from the position of the fixed "good" copy of $G_{t+1}+z$ in $x$ by $\left(n_{h}, n_{v}\right)$, $\left(n_{h}-n_{t+1}, n_{v}\right),\left(n_{h}, n_{v}-n_{t+1}\right)$, and $\left(n_{h}-n_{t+1}, n_{v}-n_{t+1}\right)$, respectively. For at least one of them, both coordinates have absolute values not larger than $n_{t+1} / 2$ and at least one coordinate has absolute value larger than $n_{t}-n_{t-1}$. 


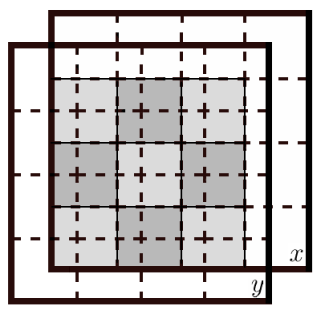

Fig. 5. The $(r-1)^{2}$ "good" copies of $G_{t+1}+z$ in $x$ are marked gray

Again, we will analyze the case where this is true for $\left(n_{h}, n_{v}\right)$; the other three cases are analogous.

There are now three further (not disjoint) cases (see Figure 6):

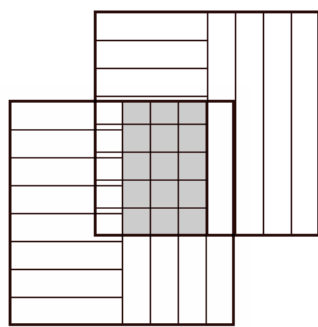

case (a)

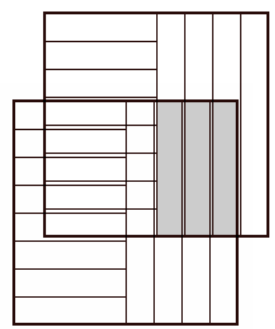

case (b)

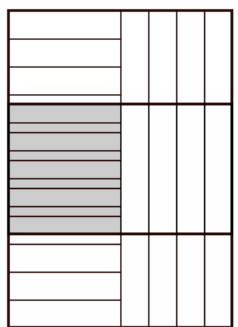

case (c)

Fig. 6. The blocks $G_{t+1}+z$ in $x$ (upper square) and $G_{t+1}+z^{\prime}$ in $y$ (lower square)

(a) $n_{t+1} / 4<n_{h}$. Then the overlap area of the left half of the copy of $G_{t+1}+z$ in $x$ with the right half of $G_{t+1}+z^{\prime}$ in $y$ occupies at least $1 / 8$ of the total area of $G_{t+1}+z$. Contributing this fraction to the rate of mixing, for the rest of case (a) we restrict our attention to this area. Here the horizontal bars of $x$ meet the vertical bars of $y$. Choose one such horizontal bar in $x$, where the "shoving vector" $\bar{h}_{i}$ is zero. Within this bar, mark all SW-corners (bottom-left corners of copies of $D_{t-1}$ ) in $y$ which fall inside copies of $D_{t-1}$ of $x$. We first need to estimate the percentage of the marked SW-corners among all SW-corners in $y$ within the selected bar. Because we are down one step in the hierarchy, we must admit copies of $D_{t-1}$ organized in $y$ in (truncated) bars which meet either orthogonal or parallel (truncated) bars in $x$ (truncation is necessary because we consider blocks $G_{t+1}+z$ and $G_{t+1}+z^{\prime}$, not just $\left.G_{t+1}\right)$. In both cases the fraction is (nearly) $2 / 3$, because if we consider a square portion of a vertical or horizontal bar in form of $D_{t-1}^{3 \times 3}$ then, in the majority of such squares, at most three copies of $D_{t-1}$ (one row or one column) have their $\mathrm{SW}$-corners in the gaps between the bars in $x$. This contributes $2 / 3$ to the rate of mixing. 
Now advance up and down by the vertical step $3 r n_{t}$. The positions of the marked SW-corners in $y$ repeat periodically, because in $y$ we are moving along a vertical bar, which is vertically $3 r n_{t}$-periodic. We mark all these repetitions too. The positioning of the horizontal bars in $x$ is only approximately vertically periodic; the copies of $D_{t-1}$ in $x$ fluctuate from their periodic positions by the nonnegative displacements $\bar{h}_{i}$ with $i$ ranging from 1 to a number larger than half the length of the vector $H$. By the properties of $H$, the values $\bar{h}_{i}$ are (nearly) equidistributed over the size of $D_{t-1}$, hence among the marked SW-corners in $y$ the fraction of nearly $1 /\left(r n_{t-1}\right)^{2}$ meet an SW-corner in $x$. For large $t$ the last fraction nearly equals $\mu\left(D_{t-1}\right)$. Thus, in case (a) we have obtained $\mu\left(\left[D_{t-1}\right] \mid T^{\bar{n}}\left[D_{t-1}\right]\right) \geq C \mu\left(\left[D_{t-1}\right]\right)$, as needed.

(b) $n_{t}-n_{t-1}<n_{h} \leq n_{t+1} / 4$. In this case the right half of the copy of $G_{t+1}+z$ in $x$ overlaps with the right half of $G_{t+1}+z^{\prime}$ in $y$ on at least $1 / 8$ of the total area. Here, vertical bars of $x$ meet vertical bars of $y$. The "worst case" arises when the horizontal shift $n_{h}$ is close to its lower bound, as then each vertical bar of $x$ overlaps mostly with the same (meaning with the same index $i$ ) vertical bar in $y$. Nevertheless, each bar of $x$ overlaps slightly with the following bar in $y$. The overlap is at least $n_{t}$ minus $n_{t-1}$ minus the maximal width of the gap between the bars. All the negative part is at most $(r+1) n_{t-1}$, which is negligible in comparison with $n_{t}$. The overlap is hence approximately $1 / 3 r$ of the width of the bar. In all other cases, bar number $i$ of $x$ overlaps even more with bar number $i+k$ for some $k \in\left[1, q_{t+1} / 2\right)$, the same for every $i$. This contributes $1 / 3 r$ to the rate of mixing and we now restrict the search to such overlap areas. Recall that we need to count the fraction of SW-corners (of $D_{t-1}$ ) in $y$ matching those in $x$.

The situation is slightly more complex than in case (a), as we will need to use the random vectors $\bar{v}_{i+k}-\bar{v}_{i}$ which are no longer nonnegative, moreover, we control their distribution only when regarded modulo $r n_{t-1}$ (see second part of Lemma 1). This is why we need to start with SW-corners in $y$ which fall into "special" copies of $D_{t-1}$ of $x$ with the property that all four corners are SW-corners (of some copies of $D_{t-1}$ ). It is easy to see that about $2 / 3$ of all copies of $D_{t-1}$ in $x$ have this property. We find an overlap area for which $\bar{v}_{i+k}-\bar{v}_{i}=\overline{0}$ and we mark the SW-corners of $y$ which fall inside the special copies of $D_{t-1}$ in $x$. The fraction of marked corners (among all corners in $y$ in the considered overlap area) is at least (slightly less than) $1 / 3$, because, as before, in the majority of squares of the form $D_{t-1}^{3 \times 3}$ in $y$, three SW-corners may fall outside the bars of $x$, three more may be inside the bars but not in the special copies of $D_{t-1}$ (see Figure 7).

This contributes $1 / 3$ to the rate of mixing. Consider one fixed marked $\mathrm{SW}$-corner in $y$. The differences between this SW-corner and all four corners 


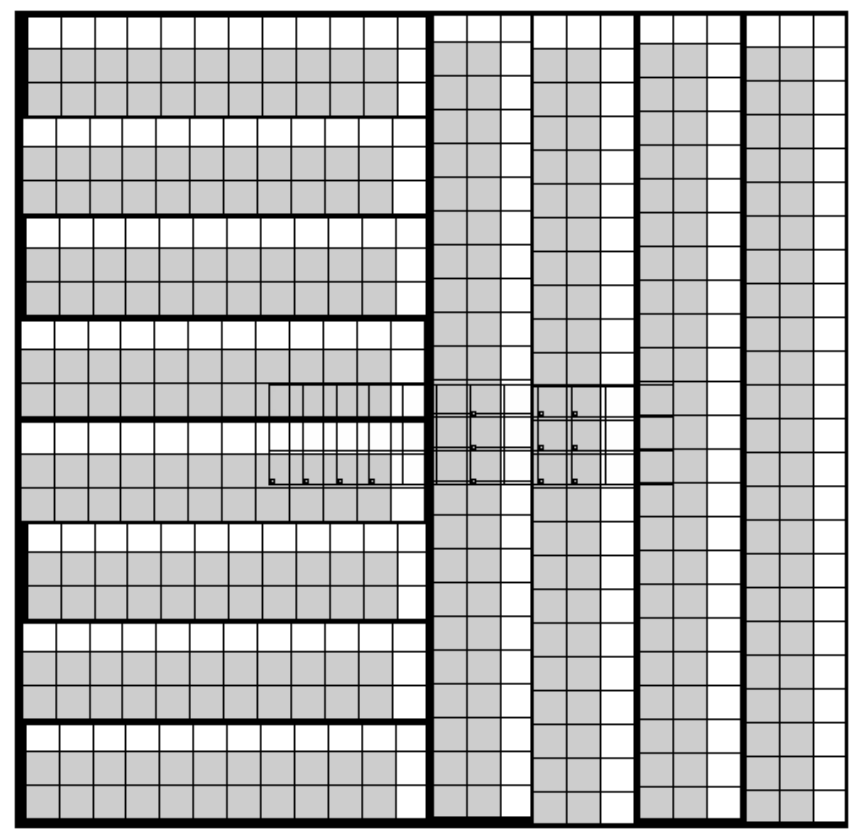

Fig. 7. The block $G_{t}$ in $x$ with its copies of $D_{t-1}$ (the picture for $G_{t}+z$ differs insignificantly; the bars are truncated at both ends). The "special" copies are shaded gray. One horizontal bar of $y$ is shown, the SW-corners falling into the special copies are marked by dots.

of the background copy of $D_{t-1}$ are all equal modulo $r n_{t-1}$, i.e., represent the same point $\bar{v}_{0}$ in $\mathbb{Z}_{r n_{t-1}} \times \mathbb{Z}_{r n_{t-1}}$. Now advance left and right by the horizontal step $3 r n_{t}$. The positions of the marked SW-corner fluctuate in $y$ by $\bar{v}_{i+k}$, while the "background" copies of $D_{t-1}$ in $x$ fluctuate by $\bar{v}_{i}$. By the properties of the random vector $V, \bar{v}_{i+k}-\bar{v}_{i}=\bar{v}_{0}\left(\bmod r n_{t-1}\right)$ approximately with frequency $1 /\left(r n_{t-1}\right)^{2}$ among all the observed corners. In all such cases the $\mathrm{SW}$-corner in $y$ matches an $\mathrm{SW}$-corner in $x$. As in the previous case, this implies $\mu\left(\left[D_{t-1}\right] \mid T^{\bar{n}}\left[D_{t-1}\right]\right) \geq C \mu\left(\left[D_{t-1}\right]\right)$, as desired.

(c) $n_{h} \leq n_{t}-n_{t-1}$ and $n_{v}>n_{t}-n_{t-1}$. This case is fully analogous to the previous one. We now examine the overlap between the left halves (which is nearly $1 / 4$ of the whole area) where the horizontal windows meet horizontal windows.

The smallest mixing rate in all above three cases is the valid mixing rate for the transformation. The proof of partial mixing is complete.

Acknowledgments. The authors wish to thank Jean-Paul Thouvenot and Valery Ryzhikov for encouragement and sharing their expertise on the subject. 
The first author acknowledges the hospitality of Laboratoire de Probabilités et Modèles Aléatoires, Université Pierre et Marie Curie, where the draft of this paper was written.

This research was supported by grant MENII 1 P03A 021 29, Poland.

\section{References}

[1] T. Downarowicz and J. Kwiatkowski, Weak Closure Theorem fails for $\mathbb{Z}^{2}$-actions, Studia Math. 153 (2002), 115-125.

[2] J. King, Commutant is the weak closure of the powers, for rank-one transformations, Ergodic Theory Dynam. Systems 6 (1986), 363-384.

[3] V. Ryzhikov, Rank, rigidity of factors, and weak closure of measure-preserving $\mathbb{Z}^{n}$ actions, Moscow Univ. Math. Bull. 63 (2008), 135-137.

Institute of Mathematics and Computer Science

Wrocław University of Technology

Wybrzeże Wyspiańskiego 27

50-370 Wrocław, Poland

E-mail: downar@pwr.wroc.pl

serafin@pwr.wroc.pl

Received June 18, 2008

Revised version March 20, 2009 